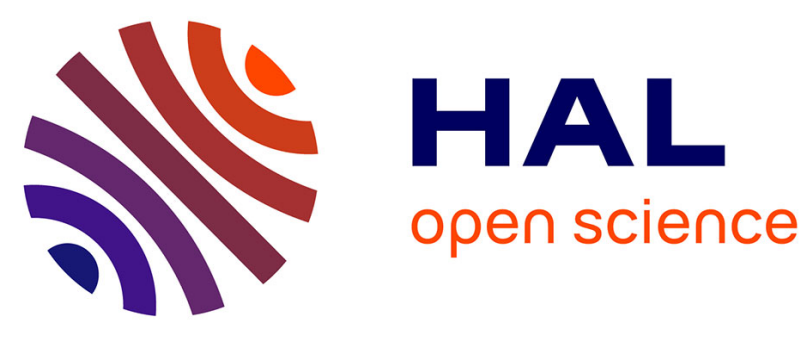

\title{
GaN light-emitting diodes with Archimedean lattice photonic crystals
}

\author{
Aurélien David, Tetsuo Fujii, Elison Matioli, Rajat Sharma, Shuji Nakamura, \\ Steven P. Denbaars, Claude Weisbuch, Henri Benisty
}

\section{- To cite this version:}

Aurélien David, Tetsuo Fujii, Elison Matioli, Rajat Sharma, Shuji Nakamura, et al.. GaN lightemitting diodes with Archimedean lattice photonic crystals. Applied Physics Letters, 2006, 88 (7), pp.073510. 10.1063/1.2168673 . hal-00869574

\section{HAL Id: hal-00869574}

\section{https://hal-iogs.archives-ouvertes.fr/hal-00869574}

Submitted on 3 Oct 2013

HAL is a multi-disciplinary open access archive for the deposit and dissemination of scientific research documents, whether they are published or not. The documents may come from teaching and research institutions in France or abroad, or from public or private research centers.
L'archive ouverte pluridisciplinaire HAL, est destinée au dépôt et à la diffusion de documents scientifiques de niveau recherche, publiés ou non, émanant des établissements d'enseignement et de recherche français ou étrangers, des laboratoires publics ou privés. 


\title{
GaN light-emitting diodes with Archimedean lattice photonic crystals
}

\author{
Aurélien David, ${ }^{\text {a) }}$ Tetsuo Fujii, ${ }^{\text {b) }}$ Elison Matioli, Rajat Sharma, Shuji Nakamura, \\ Steven P. DenBaars, and Claude Weisbuch ${ }^{\text {c) }}$ \\ Materials Department, University of California, Santa Barbara California 93106 \\ Henri Benisty \\ Laboratoire Charles Fabry de l'Institut d'Optique, Bat 50391403 Orsay, France
}

(Received 14 November 2005; accepted 21 December 2005; published online 16 February 2006)

\begin{abstract}
We study GaN-based light emitting diodes incorporating an omnidirectional photonic crystal with Archimedean lattice. Photonic bands are observed over several Brillouin zones, revealing reciprocal space symmetries and evidencing the omnidirectionality of the photonic crystal. Intensities of the diffracted bands are found to agree with the Fourier transform of the crystal lattice, and confirm its Archimedean nature. (C) 2006 American Institute of Physics. [DOI: 10.1063/1.2168673]
\end{abstract}

Photonic crystals ( $\mathrm{PhCs}$ ) have been repeatedly considered as potential candidates for light extraction from lightemitting diodes (LEDs), as they could extract the emitted light otherwise trapped inside the semiconductor due to its index contrast with air. ${ }^{1-6}$ Recently, PhCs were used as outcoupling gratings in GaN-based LEDs, operating at blue and UV wavelengths. ${ }^{7-10}$ While some enhancements in light extractions was obtained by basic lattices, the huge parameter space of $\mathrm{PhCs}$ is far from being explored and could lead to highly efficient light-extracting structures.

Among other desirable properties, a $\mathrm{PhC}$ outcoupler should be omnidirectional; that is, be able to diffract guided light incoming at any azimuthal angle. In typical secondorder gratings (with a lattice constant $a \sim \lambda / n$, where $\lambda$ is the wavelength and $n$ the index of the material), diffraction is due to the nearest neighbors of the reciprocal lattice (RL), and this property cannot be fulfilled: even with a triangular lattice, the number of nearest neighbors in the RL is only 6 . As the extraction occurs over an angular range $\sim \pm 1 / n_{\text {eff }}$ around each of these points, only $80 \%$ of all azimuthal angles are addressed in the case of $\mathrm{GaN}$ where $n_{\mathrm{eff}} \sim 2.4{ }^{11}$ High-order gratings $(a \ll \lambda / n)$ have the opposite property because many points of the RL lay in the air cone, whatever the direction of the mode. However, they generally present two drawbacks: the extraction length scales as $a$ so that an extended $\mathrm{PhC}$ is needed to extract all light, decreasing the brightness; and the number of reciprocal points in the substrate cone grows faster than in the air cone, so that more light is diffracted in the substrate.

On the other hand, omnidirectionality has also been sought as a desirable property for other $\mathrm{PhC}$ applications, especially as concerns band gaps. A number of alternative crystal lattices have been suggested, essentially to obtain band gaps of similar magnitude in all directions: Archimedean tilings, ${ }^{12}$ Penrose lattices,${ }^{13-15}$ or other quasicrystals. ${ }^{16-19}$ It is expected that such isotropic behaviors can be transposed to light-diffracting PhCs. In this letter, following the suggestion of Ref. 20, we study the use of A7-type Archimedean tilings in GaN-based LEDs.

\footnotetext{
a) Also at Laboratoire Charles Fabry de 1'Institut d'Optique, Orsay, France; electronic mail: aurelien.david@polytechnique.org

${ }^{b)}$ Also at NICP/ERATO UCSB.

c) Also at Laboratoire de Physique de la Matière Condensée, Ecole Polytechnique, 91128 Palaiseau, France.
}

The epitaxial structure used for LEDs fabrication is as follows: a $3 \mu \mathrm{m}$ thick $\mathrm{GaN}$ buffer, a $800 \mathrm{~nm}$ thick $\mathrm{Al}_{0.1} \mathrm{Ga}_{0.9} \mathrm{~N}$ layer, and a $600 \mathrm{~nm}$ thick $\mathrm{GaN}$ cap layer with a multi-quantum-well region emitting at $\lambda \sim 450 \mathrm{~nm}$ embedded at its center. LEDs are formed on the material, with a $p$-contact injection area of 100 by $100 \mu \mathrm{m}$. The AlGaN layer acts as a lower-index cladding layer, so that the GaN cap layer on top of it forms a monomode waveguide. Its presence is expected to increase the efficiency of the $\mathrm{PhC}$ grating. ${ }^{10}$

A7 patterns are then formed on top of some LEDs by electron-beam lithography, and transferred to the GaN by reactive ion etching using a $\mathrm{SiO}_{2}$ hard mask, with a depth $\sim 250 \mathrm{~nm}$. Figure 1 presents an atomic force microscope (AFM) image of the A7 pattern and displays the corresponding Fourier coefficients of the dielectric map $\widetilde{\boldsymbol{\epsilon}}(\mathbf{G})$ (which gives the coupling strength between two RL points separated by a reciprocal vector $\mathbf{G}$ ). An A7 is actually a triangular lattice with a more complex basis made of 7 "atoms" (holes here) per unit cell, all holes being separated by a distance $a$. LEDs were fabricated, incorporating PhCs with $a=190,200$, and $215 \mathrm{~nm}$. The crystal lattice is $b=a(1+\sqrt{3})$ : this is indeed a high-order crystal, where the second to seventh-nearest RL neighbors cause diffraction to air. However, due to the complex basis of the A7, a few of these RL points have the largest "photonic strength" [the largest $\widetilde{\boldsymbol{\epsilon}}(\mathbf{G})$ value], while other RL points carry little strength. Thus, the two drawbacks usually associated with high-order lattices are circumvented: the RL points responsible for diffraction to air are strong, inducing a short extraction length, while the other points are weak, diffracting little power to the substrate.
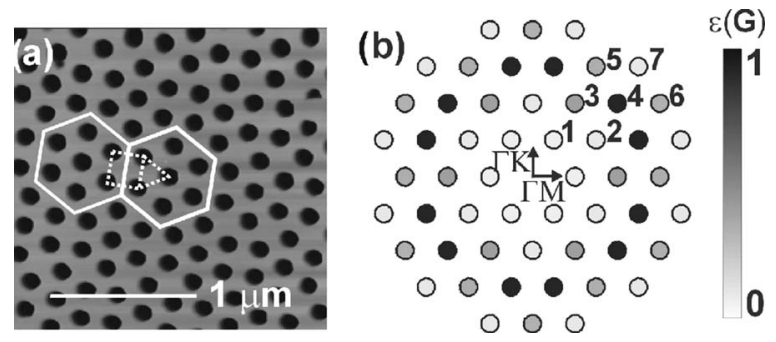

FIG. 1. (a) AFM image of an $\mathrm{A} 7 \mathrm{PhC}$ (full white line: unit cell of the crystal). (b) Fourier transform of the dielectric map $\widetilde{\boldsymbol{\epsilon}}$ (in arbitrary units) for a filling factor $f=0.3$. The RL points are indexed by their distance from the center. 


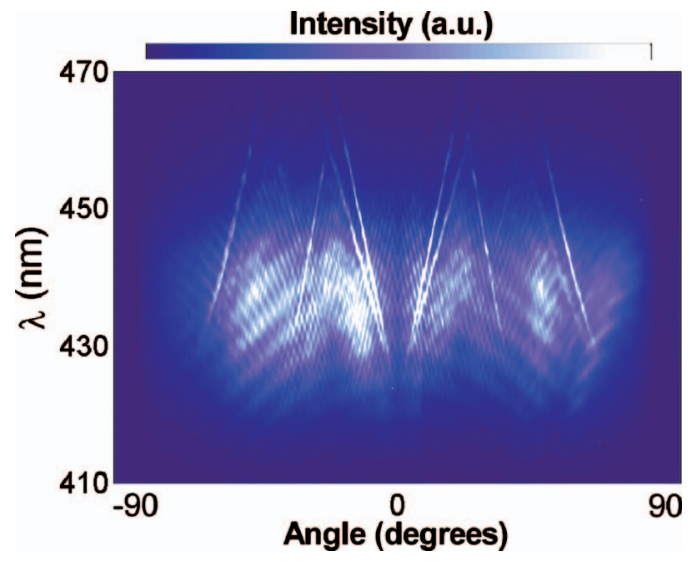

FIG. 2. (Color) Angular-resolved spectrum for a PhC with $a=190 \mathrm{~nm}$, along the $\Gamma M$ direction (TE polarization).

In order to characterize the band structure of the $\mathrm{PhCs}$, the LEDs' emission patterns $I(\lambda, \theta)$ were measured by angleresolved electroluminescence, as in Refs. 9 and 10. The angular spectra were then converted to band structures after normalization of the wavelength and angle dependence as in Ref. 9 [and using $k_{\|}=2 \pi \sin (\theta) / \lambda$ ]. In the band diagrams, the direct emission from the radiative modes of the LED and the photonic bands diffracted by the $\mathrm{PhC}$ are superimposed. Figures 2 and 3, respectively, show an original angular spectrum and band structures of LEDs measured with various periods, polarizations, and crystal directions.

The basic features of the band structure are comparable to those of Refs. 9 and 10: the GaN waveguide is strongly multimode, and each mode is folded by periodicity, giving rise to photonic bands that can be measured when they are above the light line of air. As we are observing the band structure at high frequency, the guided modes give rise to sets of bands (each of which is generated by a RL point in the air cone). One set of modes is significantly more intense than the others; this set stems from the mode guided in the $\mathrm{GaN}$ cap layer, which carries an important fraction of the total light emission. This set also proves very useful to study the band structure: it is clearly distinguishable from all the other sets (which form a quasicontinuum in this thick sample). It can easily be fitted by a simple two-dimensional $\mathrm{PhC}$ band structure calculation, leading to an effective index $n_{\mathrm{eff}}=n_{\mathrm{GaN}}-0.05$ for this set of modes [superimposed on Fig. 3(a)].

In addition to these general features, it is worth noticing that, due to the large crystal lattice, the angular measurement gives access to several Brillouin zones (BZs). For instance, at a frequency $b / \lambda=1.2$, the first $M$ point corresponds to light scattered at an angle $\theta=29^{\circ}$, and all subsequent angles correspond to measurements in the second BZ. In Fig. 3, the edges of the first $\mathrm{BZ}$ are made visible. Along the $\Gamma M$ direction, the $\mathrm{PhC}$ bands are symmetric with respect to the $\mathrm{BZ}$ edge; this is expected since the path followed in reciprocal space corresponds to $\Gamma M \Gamma$ (this symmetry is visible only for some of the bands, because others have very weak intensity). On the other hand, when measuring along the $\Gamma K$ direction farther than the first $K$ point, one actually scans the $K M$ direction of the reciprocal space: the bands are then not symmetric at the $K$ edge of the Brillouin zone. The corresponding paths in the reciprocal space are depicted in Fig. 4. It is quite satisfying that these reciprocal space symmetries are

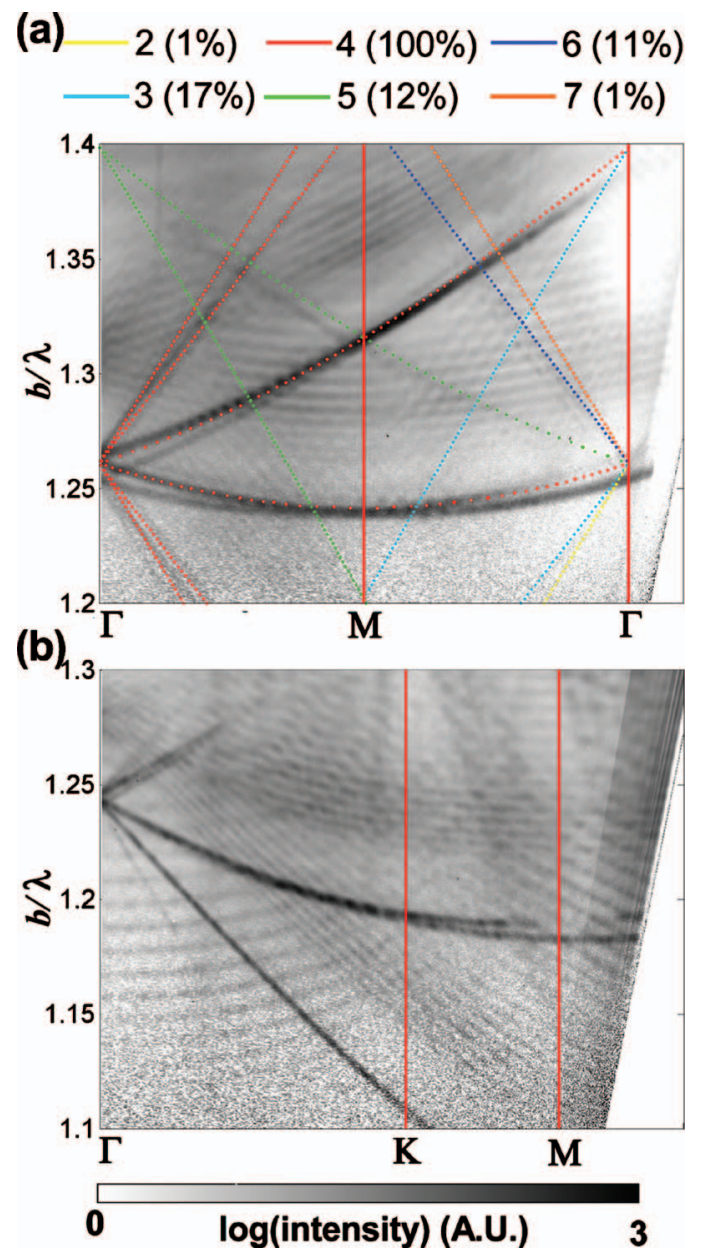

FIG. 3. (Color) Measured band structures (full red lines: Brillouin zone boundaries). (a) $a=215 \mathrm{~nm}, \Gamma M$ direction, TM polarization. Dots: Theoretical band structure of the mode guided in the cap layer. The color of the dots indicates the reciprocal lattice point responsible for diffraction (the corresponding power diffraction rates are indicated in parenthesis, in arbitrary units). (b) $a=200 \mathrm{~nm}, \Gamma K$ direction, TM polarization.

well reproduced experimentally. Moreover, the measurement confirms that the whole first $\mathrm{BZ}$ is inside the air cone, so that all guided modes can be extracted to air; this $\mathrm{PhC}$ is indeed omnidirectional.

So far, however, the mentioned features make no proof of the difference between the A7 lattice and a high-order triangular lattice with only one hole per unit cell: the dispersion diagrams of both lattices are similar-at least for such perturbative $\mathrm{PhCs}$, where the dispersion is essentially that of free photons-and the difference between the two lattices lies in the intensity of RL points.

As argued before, the coupling between RL points decreases with their distance in a triangular lattice: more precisely, $\widetilde{\epsilon}(\mathbf{G}) \sim J_{1}(G R) / G R \sim G^{-3 / 2}$ (where $J_{1}$ is a Bessel func-
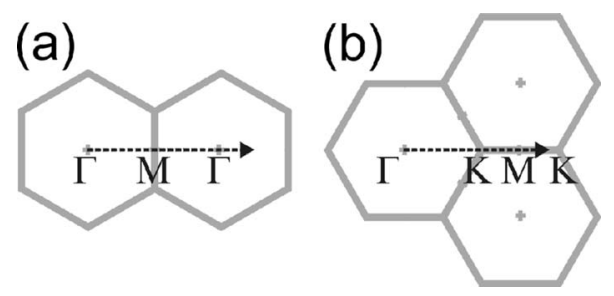

FIG. 4. Paths followed by the angular scans in the reciprocal space; (a) and (b) correspond to the spectra of Fig. 3. 
tion), and the diffracted power is proportional to $\widetilde{\epsilon}^{2} \sim G^{-3}$. In particular, for a triangular $\mathrm{PhC}$ with filling factor $f=0.3$, the power diffracted by the fourth-nearest neighbors of the RL is found to be $10 \%$ of that of the nearest neighbors. On the contrary, for an A7 lattice, constructive interference between the atoms of the unit cell redistribute most photonic strength to these RL points. In Fig. 3, the RL points responsible for diffraction are indicated for each of the calculated bands. Quite clearly, the expected trend is observed: the bands corresponding to the fourth-nearest neighbors are the most intense in the measured spectra, while some bands (bands 2 and 7) are not visible. An additional proof is brought by the overall light extraction enhancement due to the PhC. We use the same method as in Ref. 10, where the light extraction enhancement due to an LED with second-order triangular lattice $\mathrm{PhC}$ (fabricated simultaneously on the same sample) was estimated to be $\sim 70 \%$-in a numerical aperture 0.5 and at a direct current of $10 \mathrm{~mA}$. With the Archimedean lattice, the enhancement is $\sim 50 \%$. The two values are of the same order of magnitude, which indicates that the photonic strength is similar for the nearest neighbors in the triangular lattice, and the fourth-nearest neighbors in the A7 lattice.

From these elements, the collective coherent behavior of the atoms of the unit cell is quite clear: this constitutes a direct observation of the Archimedean nature of the lattice.

The total extraction enhancement measured on the A7 sample is slightly lower than that obtained with the secondorder $\mathrm{PhC}$ with a triangular lattice. A possible reason for this is the small numerical aperture of our power collection setup, which does not collect the modes diffracted at glancing angles-those are numerous for A7 lattices, according to the angular measurements. Another reason is the lack of optimization of the $\mathrm{PhC}$ parameters, which is a challenging task here due to the multiple photonic bands. Further optimization is clearly required, and preliminary theoretical results suggest that a distributed Bragg reflector should be inserted in the epitaxial structure in order to quench the remaining diffraction in the substrate by fourth-nearest neighbors of the RL.

In summary, we have fabricated and studied GaN-based LEDs with Archimedean lattice light-diffracting photonic crystal. Measurements confirm that diffraction occurs over several Brillouin zones, and the symmetry properties of the Brillouin zones are well verified experimentally. Moreover, direct proof of the Archimedean lattice behavior is observed through the intensities of the diffracted photonic bands. This approach brings complementary information to most experimental studies of nonconventional crystal lattices which focus on the band gap properties and are somehow indirect. While high-efficiency devices still call for thorough optimization, the present observations are encouraging as regards the future use of Archimedean lattices for lightdiffracting PhCs.

This work was supported by funding from the Solid State Light and Display Center at UCSB.

${ }^{1}$ M. Boroditsky, T. F. Krauss, R. Coccioli, R. Vrijen, R. Bhat, and E. Yablonovitch, Appl. Phys. Lett. 75, 1036 (1999).

${ }^{2}$ M. Rattier, H. Benisty, R. P. Stanley, J.-F. Carlin, R. Houdré, U. Oesterle, C. J. M. Smith, C. Weisbuch, and T. F. Krauss, IEEE J. Sel. Top. Quantum Electron. 8, 238 (2002).

${ }^{3}$ D. Delbeke, P. Bienstman, R. Bockstaele, and R. Baets, J. Opt. Soc. Am. B 19, 871 (2002).

${ }^{4}$ A.-L. Fehrembach, S. Enoch, and A. Sentenac, Appl. Phys. Lett. 79, 4280 (2001).

${ }^{5}$ S. Fan, P. R. Villeneuve, J. D. Joannopoulos, and E. F. Schubert, Phys. Rev. Lett. 78, 3294 (1997).

${ }^{6}$ M. Fujita, S. Takahashi, Y. Tanaka, T. Asano, and S. Noda, Science 308, 1296 (2005)

${ }^{7}$ T. N. Oder, J. Shakya, J. Y. Lin, and H. X. Jiang, Appl. Phys. Lett. 83, 1231 (2003)

${ }^{8}$ J. J. Wierer, M. R. Krames, J. E. Epler, N. F. Gardner, M. G. Craford, J. R. Wendt, J. A. Simmons, and M. M. Sigalas, Appl. Phys. Lett. 84, 3885 (2004).

${ }^{9}$ A. David, C. Meier, R. Sharma, F. S. Diana, S. P. DenBaars, E. Hu, S. Nakamura, C. Weisbuch, and H. Benisty, Appl. Phys. Lett. 87, 101107 (2005).

${ }^{10}$ A. David, T. Fujii, R. Sharma, K. McGroddy, S. Nakamura, S. P. DenBaars, E. L. Hu, C. Weisbuch, and H. Benisty Appl. Phys. Lett. 88, 061124 (2006).

${ }^{11}$ This simple geometric approach is valid if the dispersion of Bloch modes is close to that of free guided modes, which is the case for shallow-etched $\mathrm{PhCs}$ in $\mathrm{GaN}$-on-sapphire structures.

${ }^{12}$ S. David, A. Chelnokov, and J.-M. Lourtioz, IEEE J. Quantum Electron. 37, 1427 (2001).

${ }^{13}$ A. D. Villa, S. Enoch, G. Tayeb, V. Pierro, V. Galdi, and F. Capolino, Phys. Rev. Lett. 94, 183903 (2005).

${ }^{14}$ S. P. Gorkhali, J. Qi, and G. P. Crawford, Appl. Phys. Lett. 86, 011110 (2005).

${ }^{15}$ M. Notomi, H. Suzuki, T. Tamamura, and K. Edagawa, Phys. Rev. Lett. 92, 123906 (2004).

${ }^{16}$ Y. S. Chan, C. T. Chan, and Z. Y. Liu, Phys. Rev. Lett. 80, 956 (1998).

${ }^{17}$ M. E. Zoorob, M. D. B. Charlton, G. J. Parker, J. J. Baumberg, and M. C. Netti, Nature (London) 404, 740 (2000).

${ }^{18}$ P. L. Hagelstein and D. R. Denison, Opt. Lett. 24, 708 (1999).

${ }^{19}$ J. Zarbakhsh, F. Hagmann, S. F. Mingaleev, K. Busch, and K. Hingerl, Appl. Phys. Lett. 84, 4687 (2004).

${ }^{20}$ M. Rattier, H. Benisty, E. Schwoob, C. Weisbuch, T. F. Krauss, C. J. M. Smith, R. Houdre, and U. Oesterle, Appl. Phys. Lett. 83, 1283 (2003). 